\title{
Implante de marcapasos en un centro de Uruguay (2010-2019). Seguimiento y análisis
}

\author{
Dres. Gustavo Tortajada, Romina Roif, Zuly Cortellezzi, Walter Reyes Caorsi, \\ Gonzalo Varela
}

\section{Resumen}

Descripción y análisis de los procedimientos de implante de marcapasos en el servicio de electrofisiología institucional en un período de nueve años. Se analiza en forma retrospectiva un total de 1.265 procedimientos. La mediana de edad al momento del implante fue de 77 años, con predominio del sexo masculino. El bloqueo auriculoventricular y la enfermedad del nódulo sinusal fueron las indicaciones más frecuentes. El modo de estimulación predominante fue el bicameral secuencial en menores de 80 años. La mediana de seguimiento fue de 45 meses y hubo seguimiento completo en $90,7 \%$ de los pacientes. Se observó una baja tasa de complicaciones $(0,8 \%)$.

Palabras clave: $\quad$ MARCAPASOS CARDÍACO

ESTIMULACIÓN CARDÍACA

\section{Pacemaker implant in a center in Uruguay (2010-2019). Monitoring and analysis}

\section{Summary}

Description and analysis of permanent pacemakers implantation procedures at the institutional electrophysiology laboratory in a 9-year period. A total of 1265 procedures were retrospectively reviewed. Median age of implantation was 77 years, with men being the majority. Atrioventricular block and sinus node disease were the most frequent indications. Sequential dual chamber pacing was the most common programming mode in patients under 80 years old. The average follow-up was 45 months and there was a complete follow-up in $90.7 \%$ of the patients. A low complication rate was observed ( $0.8 \%$ of procedures).

Key words: $\quad$ CARDIAC PACEMAKER

CARDIAC STIMULATION

\section{Implante de marcapasso em um centro no Uruguai (2010-2019). Monitoramento e análise}

\section{Resumo}

Descrição e análise dos procedimentos de marcapassos implantados no serviço de eletrofisiológia institucional, em um período de 9 anos. Um total de 1.265 procedimentos foram revisados retrospectivamente. A mediana de idade do implante foi de 77 anos com predominância de sexo masculino. El bloqueio atrioventricular e doença do nó sinusal são as indicações mais freqüentes. O modo predominante de estimulação foi o bicameral secuencial em los menores de 80 anos. O seguimento médio foi de 45 meses e houve acompanhamento completo em 90,7\% dos pacientes. Uma baixa taxa de complicações foi observada ( $0,8 \%$ dos procedimentos).

Palavras chave: MARCAPASSO CARDÍACO

ESTIMULAÇÃO CARDÍACA 


\section{Introducción}

El implante de marcapasos (MCP) es una técnica probada que se practica de forma rutinaria desde la década de 1960 y ha contribuido a descender la morbimortalidad vinculada a los trastornos de la conducción y generación del impulso eléctrico cardíaco. El progreso en el conocimiento de estas patologías, sumado a los avances tecnológicos, han aumentado las indicaciones de estimulación cardíaca, haciéndola cada vez más fisiológica y segura para el paciente ${ }^{(1)}$.

En Sudamérica los registros o datos publicados sobre estimulación cardíaca definitiva son escasos. Uruguay no es la excepción, ya que no contamos con datos recientes publicados sobre las características de los pacientes a los que se les implanta un MCP, tipo de dispositivo, longevidad o vida útil del mismo, ni las complicaciones del procedimiento y su seguimiento.

En nuestro país estos implantes son financiados por un sistema de cobertura integral llamado Fondo Nacional de Recursos $(\mathrm{FNR})^{(2)}$, que financia tratamientos farmacológicos y técnicas de alto costo. La cobertura alcanza a toda la población, no existiendo grandes limitaciones en las autorizaciones en lo que respecta a los implantes de MCP, siempre que se indique según las pautas nacionales e internacionales. La tasa de implantes de MCP convencionales en Uruguay en 2017 fue de 403 unidades/millón de habitantes, y la de terapia de resincronización cardíaca de baja energía (TRC-P) fue de 17 unidades/millón ${ }^{(2)}$.

El objetivo de este trabajo es analizar la experiencia durante un período de nueve años en implantes de MCP y TRC-P en un servicio de electrofisiología de Uruguay. Se evaluaron características de los pacientes, tipo de dispositivo y su longevidad, las complicaciones del procedimiento y su evolución, y se compararon con registros internacionales.

\section{Material y método}

Se trata de un estudio observacional, retrospectivo, descriptivo, que analiza los implantes de MCP realizados entre el 1ํ de mayo de 2010 y el 30 de abril de 2019 en el Servicio de Electrofisiología de Casa de Galicia, Montevideo, Uruguay. Se creó una base de datos informatizada en la cual se recolectaron retrospectivamente las variables a partir de las historias clínicas. El número total de implantes en el país y de los pacientes fallecidos se obtuvo de la base informática del $\mathrm{FNR}^{(2)}$. Actualmente, se realizan en el servicio, aproximadamente 200 procedimientos vinculados a MCP al año, recibiendo pacientes de todo el país, tanto de la salud pública como de la privada.

El servicio cuenta con una sala de electrofisiología de uso exclusivo, con disponibilidad continua
¿Qué aporta este estudio al conocimiento actual?

- El implante de marcapasos es un procedimiento habitual y muy seguro en la actualidad.

- Se describen los resultados de un centro nacional con esta técnica, incluyendo un gran volumen de pacientes y la descripción detallada del protocolo utilizado. La información aportada puede ser de beneficio para los profesionales que realizan o realizarán este procedimiento.

para realizar implantes de MCP. El volumen de procedimientos vinculados a dispositivos (200-300/ año) puede considerarse como medio-alto ${ }^{(3)}$, al que deben sumarse los procedimientos de ablación por catéter y otros. En la figura 1 se muestra el progresivo aumento en el número de procedimientos/año, a excepción de 2015, con un descenso temporal debido a reformas.

En el período analizado el equipo se conformó por dos cardiólogos implantadores entrenados, según la definición de las pautas internacionales (al menos 400 implantes y más de 100 implantes/ año) ${ }^{(4,5)}$. Ambos profesionales realizan el mismo volumen anual. Además, durante el período se contó con un cardiólogo en entrenamiento y un residente de tercer año con participación activa y supervisada. El personal no médico (enfermería y técnicos) pertenece en forma exclusiva al servicio.

Los generadores y electrodos implantados fueron de cuatro proveedores de dispositivos: Boston Scientific $^{\circledR}$, Biotronik $^{\circledR}$, Medtronic $^{\circledR}$ y St. Jude Me$\operatorname{dical}^{\circledR}$.

Se utilizó un protocolo estricto para la prevención de complicaciones infecciosas desarrollado en conjunto con el comité de infecciones, diferenciando implantes de urgencia y de coordinación. En la coordinación se indican al paciente dos a tres baños con jabón de clorhexidina al $4 \%$ en las 24 horas previas al procedimiento. Los pasos posteriores no se diferencian para los procedimientos de coordinación y urgencia. El lavado se reitera en la antesala, además de la aplicación de mupirocina nasal. No se realiza rasurado. De existir vello abundante sobre el sitio quirúrgico, se recorta con máquina eléctrica. Se administra antibiótico profiláctico 30 minutos antes del inicio del implante, habitualmente una cefalosporina de primera generación por vía endovenosa. Una vez en sala, se procede a la asepsia de la piel con clorhexidina alcohólica al 4\%, colocación de campos y batas descartables, y campo adhesivo para el sitio quirúrgico. Una vez preparado el campo operatorio y la mesa de instrumental, el operador se cambia de guantes, lo cual 


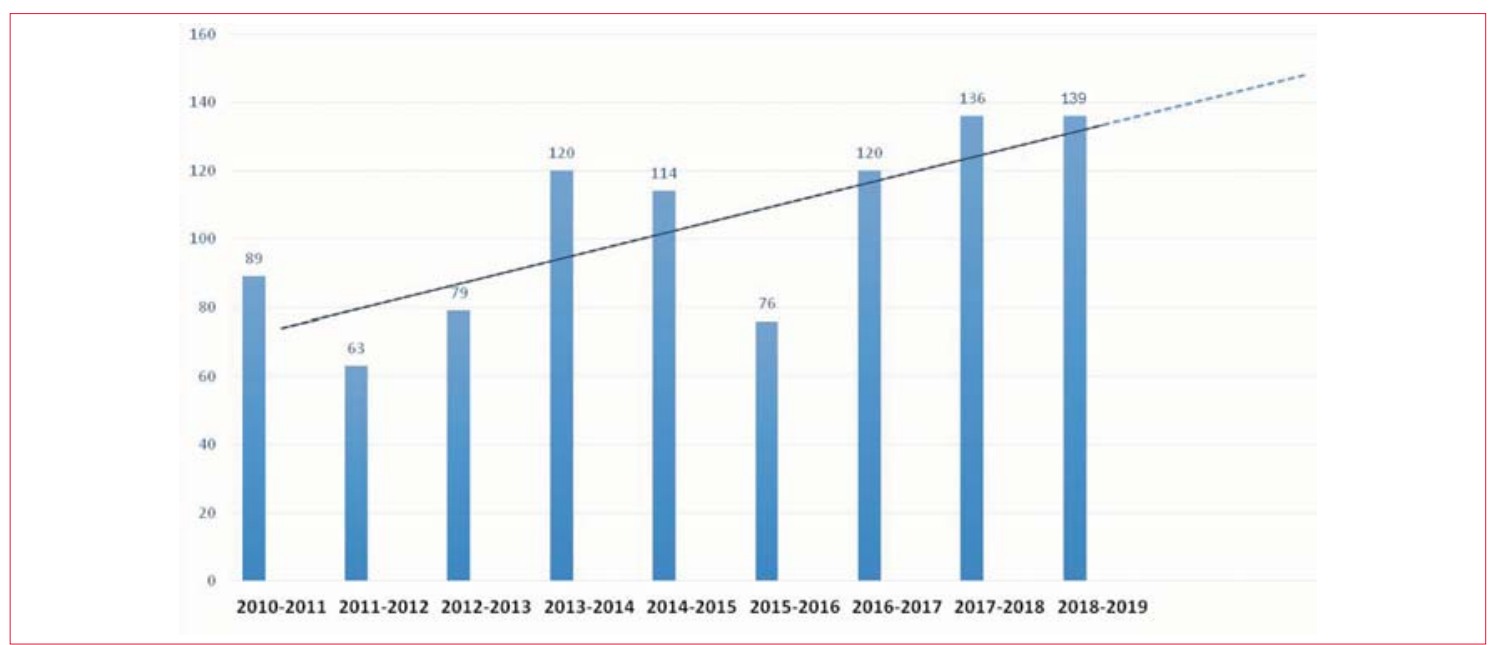

Figura 1. Evolución del volumen anual de primo-implantes.

reitera si el procedimiento dura más de $40 \mathrm{minu-}$ tos, y obligatoriamente previo a la manipulación del generador. El embalaje del MCP solo es abierto en el momento de conectarlo a los electrodos. Durante el tiempo de posicionamiento y medidas de los electrodos, se deja una gasa embebida en solución fisiológica y antibióticos (cefalosporina de primera generación) en el bolsillo del MCP. Previo a alojar el generador, se realiza lavado por arrastre con suero fisiológico y antibiótico, revisándose minuciosamente la hemostasia. Luego del cierre por planos, se realiza cura plana con uso de mupirocina en crema sobre la incisión. Esta se ocluye con campo adhesivo, el cual se mantiene por cinco a siete días. Este protocolo se resume en la tabla 1. Los pacientes se controlan previamente al alta entre los 10 a 14 días del implante, cuando se extraen los puntos de sutura y se controla la herida, luego a los 3, 6 y 12 meses. Posteriormente, el control se realiza de forma anual hasta que comienza a declinar la batería, desde entonces los controles se vuelven más frecuentes, hasta el recambio.

\section{Resultados}

Se realizaron 1.265 procedimientos: 936 primo-implantes, 295 recambios, 14 reposicionamientos de electrodos, 8 implantes de electrodos por disfunción, 7 exploraciones y reparaciones de la bolsa, 5 extracciones y explantes (figura 2). Se observó un aumento del número de primo-implantes en el período analizado, registrándose 139 primo-implantes/año en el último año (figura 2). La mediana de seguimiento fue de 45 meses y se logró el seguimiento completo en el $90,7 \%$ de los pacientes.
Tabla 1. Protocolo de prevención infecciones en los implantes.

1) Urgencia/Coordinación

2) Descartar infecciones activas sistémicas y lesiones de piel en zona de operación

3) Baños en domicilio con clorhexidina jabonosa al $4 \%$

4) Corte de vellos con máquina (no rasurado)

5) Aplicación de mupirocina $2 \%$ en región nasal

6) Cefradina $1 \mathrm{~g}$ intravenoso 30 minutos previos al procedimiento. Si es alérgico, vancomicina $1 \mathrm{~g}$ intravenoso 30 minutos previos al procedimiento

7) Lavado de manos según protocolo quirúrgico

8) Asepsia de zona del tórax con clorhexidina alcohólica al 4\%, mínimo dos aplicaciones

9) Uso de material descartable y campos adhesivos

10) Cambio de guantes en cada etapa dentro de sala y cada 40 minutos

11) Cambio de guantes previo a la manipulación del nuevo dispositivo

12) Protección del bolsillo de MCP con gasas embebidas en antibiótico durante implante de electrodos.

13) Lavado del bolsillo de MCP por arrastre con SF + antibiótico (cefalosporina)

14) Cierre del bolsillo de MCP por planos

15) Curación plana de herida con mupirocina $2 \%$ en crema

16) Oclusión con campo adhesivo estéril por 5-7 días

MCP: marcapasos; SF: suero fisiológico. 


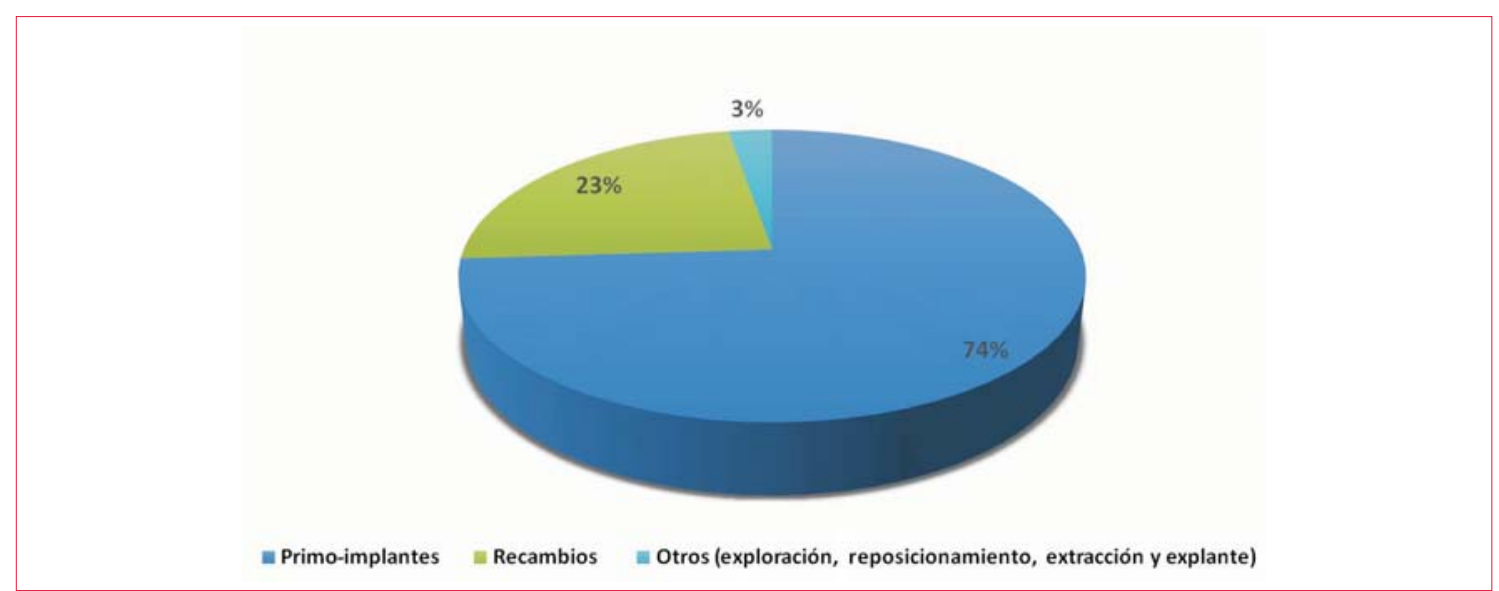

Figura 2. Total de procedimientos (\%).

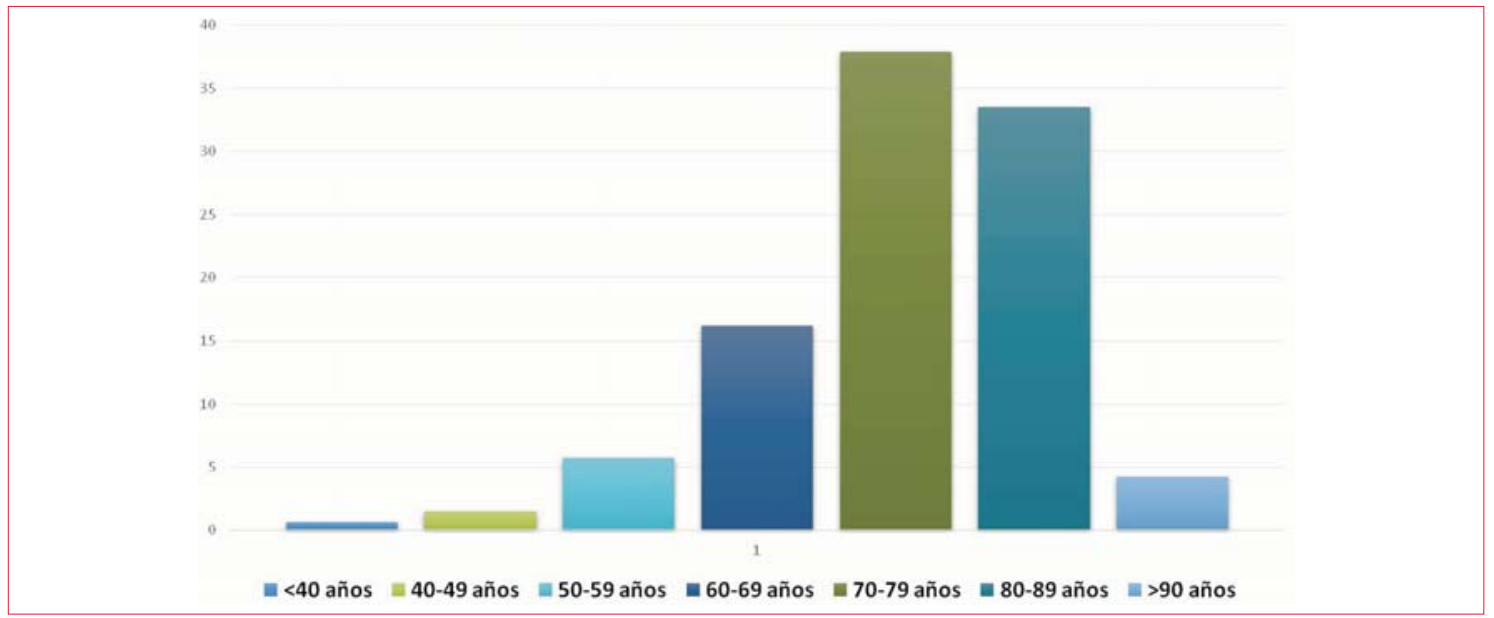

Figura 3. Primo-implantes según rango etario (\%).

\section{Edad y sexo}

La mediana de edad en los primo-implantes fue de 77 años (16-99) y en los recambios de generador de 79 años (24-98). En el análisis por décadas, el mayor número de implantes se realizó entre los 70-79 años (37,9\%), seguido en forma decreciente por las décadas entre 80-89 (33,5\%), 60-69 (16,2\%) y 50-59 $(5,8 \%)$. En pacientes mayores de 90 años los implantes fueron $4,3 \%$ y en menores de 50 años $2,0 \%$ (figura 3).

Hubo predominio de pacientes del sexo masculino, $736(58,2 \%)$ frente a 529 de sexo femenino $(41,8 \%)$. Esta diferencia se mantuvo al analizar los primo-implantes versus los recambios. Recibieron un primo-implante 549 hombres $(58,7 \%)$ y $387 \mathrm{mu}$ jeres (41,3\%); se realizó recambio del dispositivo en 168 hombres $(56,9 \%)$ y en 127 mujeres $(43,1 \%)$.

\section{Diagnóstico}

El diagnóstico más común que motivó el implante fue el bloqueo auriculoventricular (BAV) en 49,9\%

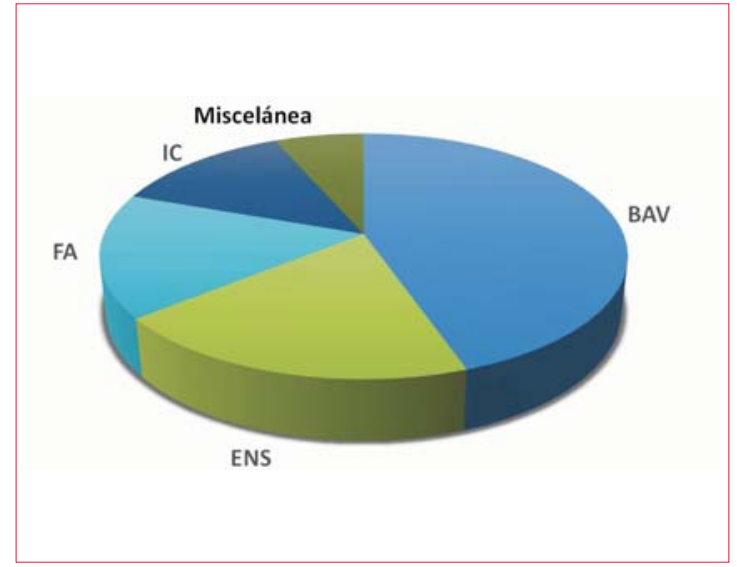

Figura 4. Indicación de primo-implantes. IC: insuficiencia cardíaca; BAV: bloqueo auriculoventricular; ENS: enfermedad del nódulo sinusal; FA: fibrilación auricular.

de los casos, a expensas de BAV de alto grado (44,7\%) y fibrilación auricular (FA)/flutter asociado a BAV de tercer grado (5,2\%). La enfermedad del nódulo sinusal (ENS) es el segundo diagnóstico más 


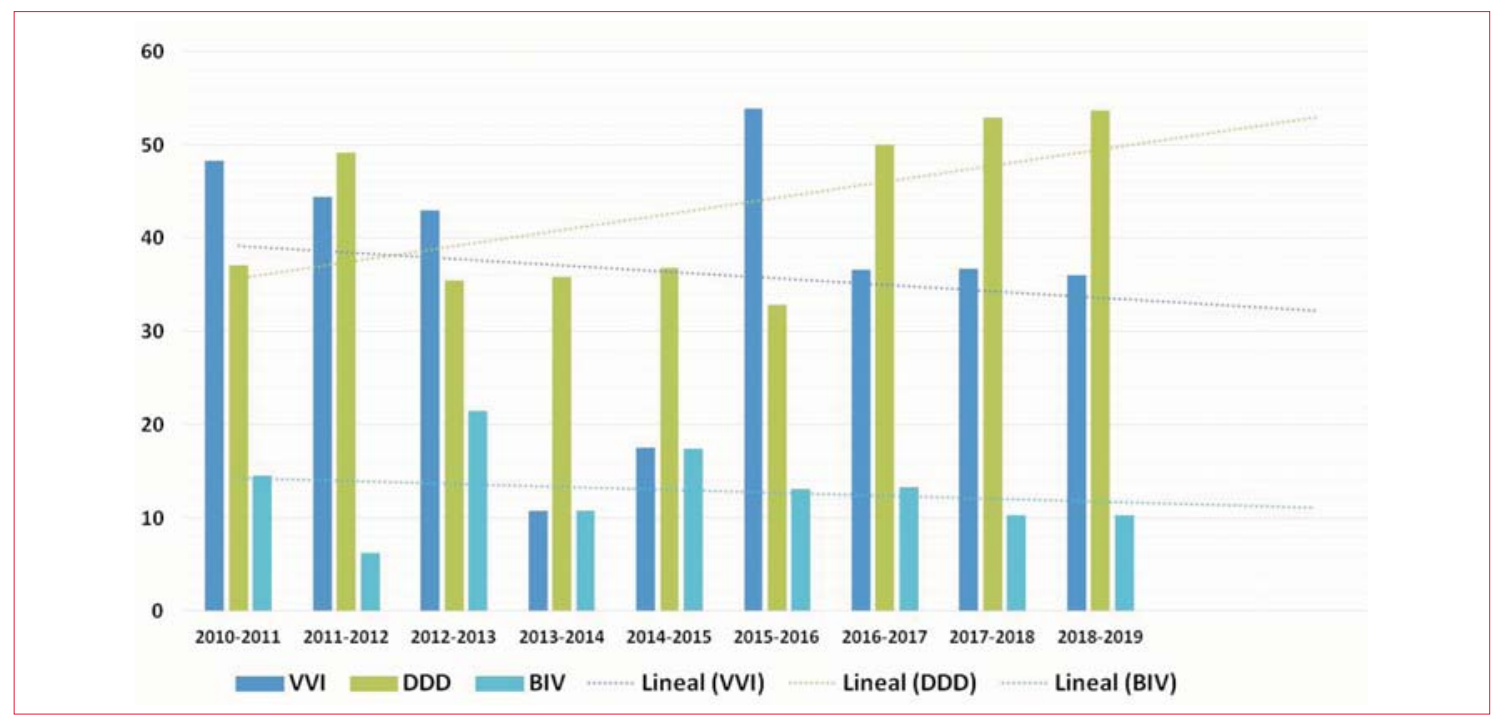

Figura 5. Evolución del modo de estimulación (\%). VVI: ventricular; DDD: auriculoventricular; BIV: biventricular.

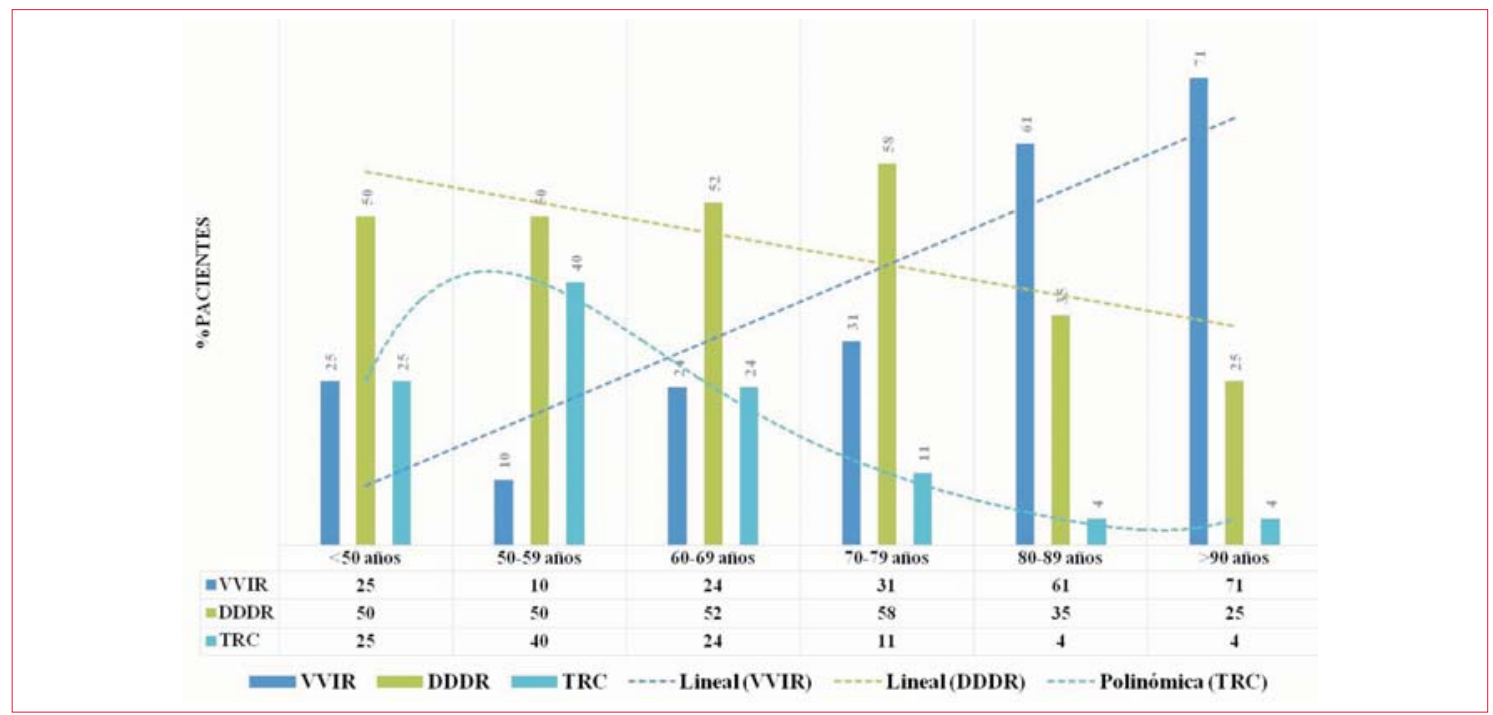

Figura 6. Modo de estimulación según franja etaria (\%).

frecuente, con el 30,5\% de los casos, incluyendo la FA con alta tasa de BAV (11,5\%). El bloqueo de rama o trastorno de conducción intraventricular representó el 3,6\% de los casos. La hipersensibilidad del seno carotídeo correspondió a $0,4 \%$. Los pacientes con insuficiencia cardíaca e indicación de resincronización representaron el 12,3\% del total. En $3,7 \%$ no se especificó la causa del implante (figura 4).

\section{Consideraciones del implante}

El 97,1\% de los implantes se realizó en la región subclavicular izquierda, y en todos los casos se confeccionó la bolsa para el generador a nivel supraponeurótico del pectoral mayor. El acceso vascular más utilizado fue por punción de la vena subclavia izquierda (91,3\%), seguido de disección de vena cefálica izquierda $(5,8 \%)$, subclavia derecha $(2,4 \%)$, cefálica derecha $(0,4 \%)$ y axilar izquierda $(0,2 \%)$. En seis casos $(0,6 \%)$ se evidenció una vena cava superior izquierda persistente. En uno de los casos se conocía previamente esta variante anatómica debido al intento fallido anterior de implante de sistema TRC-P, que se pudo realizar exitosamente por esta vía $^{(6)}$.

\section{Modo de estimulación}

En el período se implantaron 43,5\% marcapasos unicamerales (VVIR) y 43,3\% bicamerales (DDDR) (figura 5). El 12,3\% de los implantes fueron TRC-P. Este número se ha mantenido constante en el tiempo, aunque el servicio ha aumentado el número de 


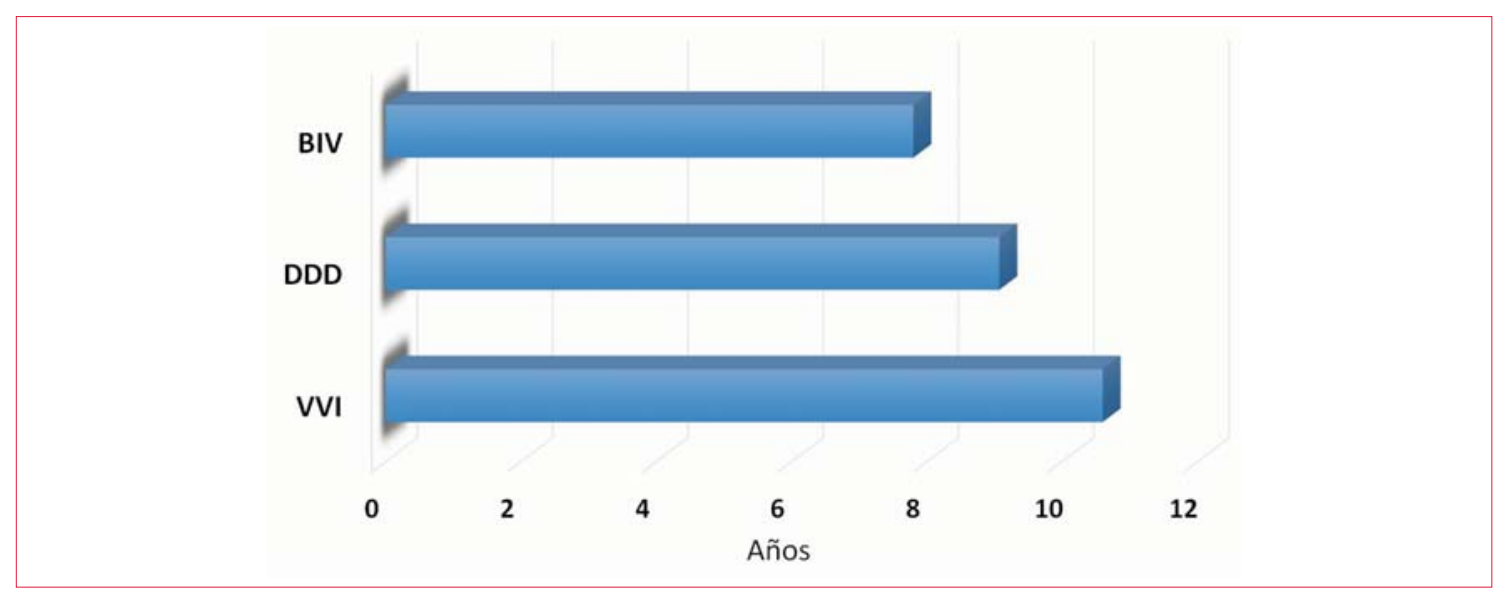

Figura 7. Longevidad promedio según modo estimulación. VVI: ventricular; DDD: auriculoventricular; BIV: biventricular.

dispositivos TRC-P a expensas del cardiodesfibrilador con resincronización en prevención primaria (no incluidos en este análisis).

La edad condicionó el modo de estimulación elegido. En los pacientes octogenarios el modo más frecuentemente indicado fue VVIR, independientemente del ritmo de base. En cambio, en los menores de 80 años predominó la estimulación DDDR y TRC-P (figura 6).

Se implantaron un total 1.577 electrodos, 936 en ventrículo derecho, 526 a nivel auricular derecho y 115 a nivel del ventrículo izquierdo. Para la estimulación auricular y ventricular derecha se utilizaron en todos los casos electrodos de estimulación bipolar. Para el ventrículo izquierdo 94,5\% de los electrodos fueron de estimulación bipolar; al 3,7\% se le implantó un electrodo unipolar, y solo $1,8 \%$ de los pacientes se refirieron a cirugía para implante del electrodo por imposibilidad de lograr un sitio adecuado y estable a través del seno coronario; $62 \%$ de los electrodos auriculares fueron de fijación activa. La mediana de la onda $\mathrm{P}$ durante el implante fue de $3,1 \mathrm{mV}$ y la mediana del umbral de estimulación auricular medido a $0,5 \mathrm{~ms}$ de ancho de pulso fue de 0,8 V. El 95,3\% de los electrodos ventriculares fueron de fijación activa; la mediana de la onda $\mathrm{R}$ durante el implante fue de $10,2 \mathrm{mV}$, y la mediana del umbral de estimulación ventricular medido a $0,5 \mathrm{~ms}$ de ancho de pulso fue de $0,6 \mathrm{~V}$.

\section{Recambios}

Se realizaron un total de 295 procedimientos de recambio de generador, 290 vinculados al agotamiento normal de la batería, tres por disfunción de los electrodos y dos para cambio de modo de estimulación (uno a DDDR y otro a TRC-P).

La mediana al tiempo de recambio fue de 8,9 años. Se observaron diferencias en la longevidad del generador según el modo de estimulación. En modo
VVIR fue de 10,6 años, mientras que en modo DDDR fue 9,1 años y en modo TRC-P 7,8 años (figura 7). La longevidad de los dispositivos resultó acorde a la estimada por los fabricantes.

\section{Complicaciones}

Se registraron cuatro infecciones $(0,3 \%)$, dos en recambios y dos en primo-implantes; cinco neumotórax normotensivos y un taponamiento cardíaco, lo que representa un total de $0,8 \%$ de complicaciones mayores. Se realizaron 14 reintervenciones para reposicionamiento de electrodos $(1,5 \%$ de los pacientes y $0,9 \%$ de los electrodos implantados). No hubo muertes relacionadas con estas complicaciones. No se registraron en la base de datos complicaciones menores (definidas como aquellas que no requirieron procedimientos adicionales).

\section{Mortalidad}

Durante todo el período de estudio se registraron 379 pacientes fallecidos $(29,7 \%)$. Cuatro pacientes murieron al mes del implante (0,3\%), 96 a los seis meses $(7,6 \%)$ y 214 al año (16,9\%) (figura 8). No se registraron muertes durante el procedimiento ni en las primeras 24 horas. No disponemos de la causa de defunción de la mayoría de los pacientes, solamente la fecha. La media de edad al fallecimiento fue de 79 años.

\section{Discusión}

Este trabajo describe la experiencia en implantes, recambios y seguimiento de MCP realizados durante un período de nueve años en el Servicio de Electrofisiología de Casa de Galicia, Uruguay.

Los datos obtenidos acerca de la edad y el porcentaje de sexo masculino se corresponden con series internacionales ${ }^{(7,8)}$. El registro del electrocardiograma (ECG) de superficie se describió adecuadamente en la 


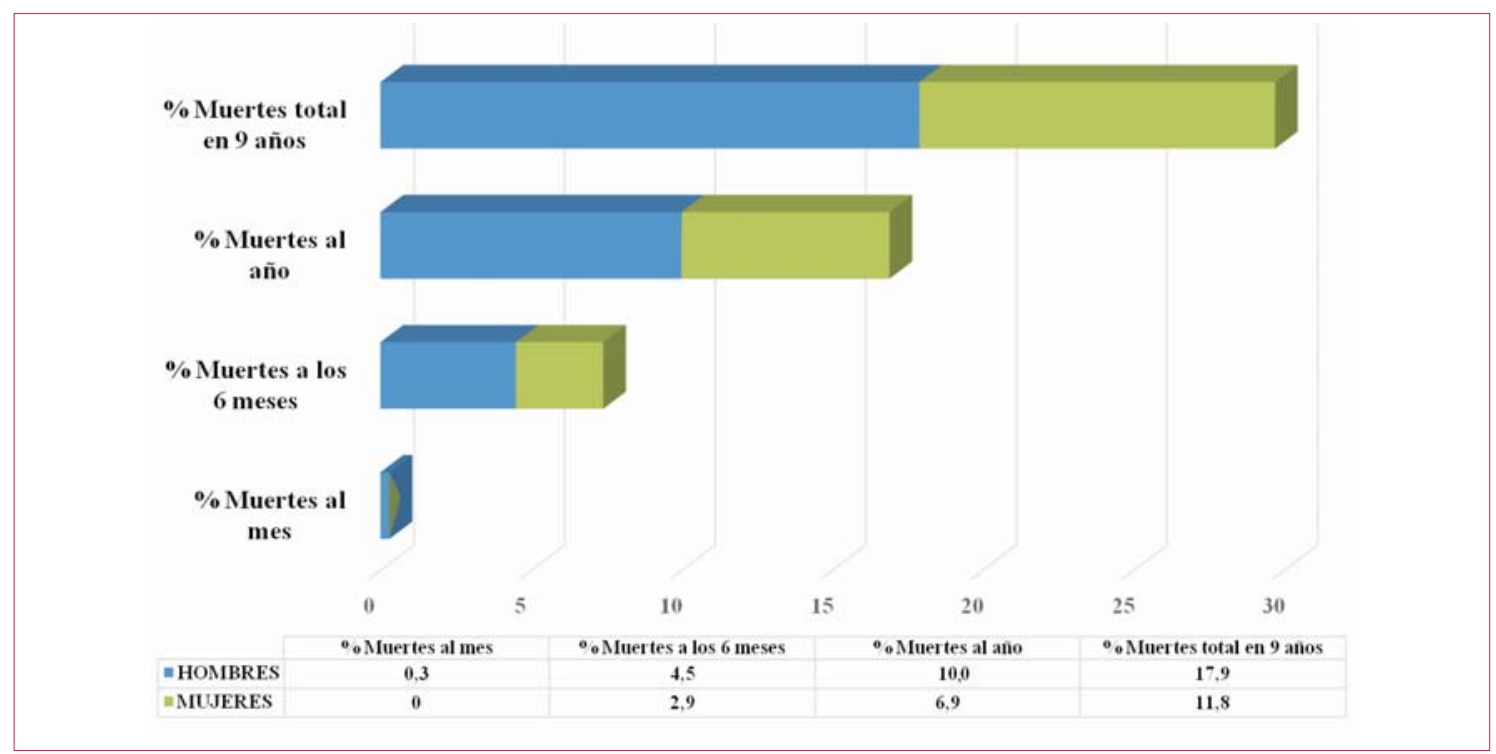

Figura 8. Mortalidad en distintos momentos del seguimiento.

mayoría de los casos (96,3\%). Al igual que en los registros danés y español, la principal causa de implante de MCP es el BAV, no discriminando en nuestro caso el tipo de bloqueo ${ }^{(7,8)}$. La segunda causa de implante de MCP es la ENS. De cualquier manera, el porcentaje de implantes por BAV es menor, y el debido a ENS es mayor que el reportado con datos oficiales del FNR para nuestro país en la encuesta mundial publicada en 2011 por el Dr. Mond (62\% BAV y 15\% ENS), que corresponden al año calendario $2009^{(9)}$. De acuerdo con esto, en la mayoría de los países europeos, la ENS es la primera causa de implante y nuestras cifras parecerían indicar una progresión en ese sentido.

El modo de estimulación utilizado se dividió equitativamente entre DDDR (43,3\%) y VVIR $(43,5 \%)$. Esta distribución es similar a la observada en el Registro Español de Marcapasos del año $2018^{(7)}$, que reporta $39,9 \%$ de modo VVIR, cifra que se ha mantenido en los últimos nueve años. Son diferentes los datos del registro danés de MCP y cardiodesfibriladores, donde se reporta $24,4 \%$ de estimulación en modo $\operatorname{VVIR}^{(8)}$. Los porcentajes registrados en nuestra casuística de uso de modo VVIR son mayores (43,5\% vs $36 \%$ ), y los de modo DDDR menores (43,3\% vs 52\%) que los reportados para el global de los países en 2009 en la encuesta mundial mencionada $^{(9)}$. En la región, a modo de ejemplo, Argentina reportó 60\% de modo VVIR y, por el contrario, Brasil reportó 70\% de modo DDDR diez años atrás. Chile y Perú reportaron una significativa predominancia del uso del modo VVIR, 70\% y $73 \%$ respectivamente ${ }^{(9)}$.

El elevado porcentaje de MCP VVIR en nuestra población podría explicarse por la alta incidencia de pacientes con FA con alta tasa de bloqueo (20\%).
Además, en los pacientes mayores de 80 años se priorizó el modo VVIR luego de una evaluación clínica completa, independientemente del ritmo de base. En cambio, en los menores de 80 años los modos de estimulación DDDR y TRC-P son los elegidos en más del $80 \%$ de los casos (figura 6). En los últimos años existe una marcada tendencia al aumento de la estimulación en modo DDDR (figura 5). En nuestro país el implante de cardiodesfibrilador en prevención primaria no está cubierto regularmente por el sistema asistencial (limitaciones, según las indicaciones internacionales). Este hecho podría generar un sesgo en el número de pacientes con sistema TRC-P.

No se usó el modo de estimulación bicameral con electrodo único (modo VDD), ni se implantaron MCP unicamerales auriculares (modo AAIR). Destacamos también el alto índice de éxito de implantes de electrodos en el ventrículo izquierdo por vía endocavitaria, con solo $1,8 \%$ de pacientes derivados a cirugía para implante de electrodo epicárdico.

El seguimiento de nuestros pacientes se realizó a través de entrevistas periódicas presenciales programadas, o excepcionalmente a requerimiento del médico tratante o del paciente. Destacamos el alto índice de adherencia a los controles, logrando un seguimiento adecuado en el 90,7\% de los casos. En nuestro país no está disponible el seguimiento remoto de los dispositivos.

Se registró una baja tasa de complicaciones mayores. Quizás esto pueda deberse a la protocolización estricta de la operativa de la sala y de sus características de funcionamiento, a la técnica y metodología uniforme del procedimiento y a la experiencia de los operadores. En registros internacionales se describe una tasa de complicaciones mayores de $1 \%$ - $2 \%(7,10,11)$ 
para operadores entrenados y en centros de alto volumen ( $0,8 \%$ en nuestro estudio).

El abordaje más utilizado fue la punción de la vena subclavia izquierda. Destacamos la baja incidencia de neumotórax como complicación de esta técnica (0,39\% de los procedimientos). De cualquier manera, actualmente estamos protocolizando la punción axilar guiada por radioscopia, dados los beneficios potenciales que han sido reportados con esta técnica(12).

Se registraron cuatro pacientes con infección del sistema que obligó a extracción o explante (dos primo-implantes y dos recambios). En ambos primo-implantes se comprobó que eran portadores nasales de Staphylococcus aureus meticilino sensible; además, uno de ellos se encontraba cursando un posoperatorio de sustitución valvular aórtica. De los dos pacientes con infección luego de un recambio, uno era portador de una enfermedad cutánea (pénfigo), y el restante sufrió una lesión accidental de piel con el bisturí eléctrico, con posterior erosión y contaminación profunda del bolsillo. El patógeno más frecuente fue Staphylococcus aureus meticilino sensible, salvo en el último caso, que cultivó Staphylococcus epidermidis. A partir de estos casos se inició el uso rutinario de mupirocina nasal (previamente en casos seleccionados) y se realizaron cultivos nasales de todo el personal. No hubo reporte de casos de endocarditis infecciosa en nuestra cohorte.

El recambio de generador no es un procedimiento banal y conlleva un mayor riesgo de infección ${ }^{(13)}$. El riesgo de infección, a pesar de ser bajo en nuestra experiencia, fue tres veces mayor que en el primo-implante $(0,67 \%$ vs $0,21 \%)$. En una serie elaborada en Estados Unidos, sin embargo, se reportó una tasa de infecciones similar en recambios y primo-implantes ${ }^{(9)}$.

De cualquier manera, estos datos apoyan la importancia de la oportunidad y la toma de decisiones previo al recambio de generador y cuando se considera un up-grade a sistemas más complejos.

En nuestra serie, 98,3\% de los recambios se realizaron por agotamiento normal del generador con una media de tiempo al recambio de 8,9 años. Observamos diferencias esperadas según el modo de estimulación, siendo el modo VVIR el de mayor duración, independientemente de la marca del generador. La longevidad de la batería según la marca y modelo del generador fue acorde a lo informado por el fabricante ${ }^{(14)}$. No contamos con datos de longevidad acorde al porcentaje de estimulación/sensado, ni al número de funciones encendidas del MCP.
Es importante destacar que no hubo muertes relacionadas con los procedimientos. Tampoco fue necesario utilizar marcapasos transitorios en los pacientes en los que se debió realizar la extracción del sistema.

\section{Fortalezas y debilidades}

A pesar de tratarse de un análisis retrospectivo y unicéntrico sobre una técnica bien establecida, aporta datos básicos importantes en un país y región donde este tipo de información es escasa. El análisis confirma la seguridad y eficacia de este tipo de procedimientos. Además, las dificultades y falencias encontradas en la recolección de los datos, han servido de base para el desarrollo de una plataforma informática con posibilidad de acceso remoto que permitirá optimizar la atención y el seguimiento de los pacientes.

Durante el período analizado se logró registrar los datos de todos los pacientes correspondientes a edad, sexo, tipo de procedimiento, característica de los electrodos, marca y modelo de los generadores, y los referentes a las complicaciones mayores. Sin embargo, fue una limitante la falta de reporte sistemático de la presentación clínica inicial, antecedentes personales pertinentes y tampoco fue registrado el sitio de implante de los electrodos, no permitiendo sacar conclusiones al respecto.

Existió dificultad en el análisis debido a la carencia de datos en algunas historias clínicas (no digitalizadas). Las variables del estudio fueron recogidas retrospectivamente desde las historias clínicas. No se registraron sistemáticamente los síntomas que motivaron la consulta y posterior implante, ni las complicaciones menores.

\section{Conclusiones}

Este es el primer estudio con seguimiento a largo plazo de implante de MCP definitivo en Uruguay. Los hallazgos respaldan la seguridad y eficacia en la práctica clínica de los procedimientos de implante y recambio de MCP, con un bajo índice de complicaciones. Destacamos el alto porcentaje de adherencia de los pacientes al tipo de seguimiento.

Pensamos que este análisis será una herramienta clave en el fortalecimiento de un sistema de trabajo y permitirá la corrección de falencias para optimizar los resultados. 
Gustavo Tortajada, https://orcid.org/0000-0002-95228299. Idea original, diseño, recolección de datos, análisis, corrección, comunicación con los editores.

Romina Roif, https://orcid.org/0000-0001-6531-587X Diseño, recolección de datos, análisis, corrección.

Zuly Cortellezzi, https://orcid.org/0000-0003-

0123-8101. Recolección de datos, análisis, corrección.

Walter Reyes Caorsi, https://orcid.org/0000-0002-

4670-5765. Corrección.

Gonzalo Varela, https://orcid.org/0000-0002-6476-

3416. Recolección de datos, análisis, corrección.

Este artículo fue aceptado para su publicación por: Editor jefe Dr. Gerardo Soca.

\section{Bibliografía}

1. Hauser RG, Hayes DL, Kallinen LM, Cannon DS, Epstein AE, Almquist AK, et al. Clinical experience with pacemaker pulse generators and transvenous leads: an 8-year prospective multicenter study. Heart Rhythm 2007;4(2):154-60. doi:10.1016 j.hrthm.2006.10.009

2. Fondo Nacional de Recursos [Internet]. Montevideo: FNR; 2019 [consulta $30 \mathrm{Abr} 2020$ ]. Disponible en: http://www.fnr.gub.uy/marcapasos

3. Todd D, Bongiorni MG, Hernandez-Madrid A, Dagres N, Sciaraffia E, Blomström-LundqvistC, et al. Standards for device implantation and follow-up: personnel, equipment, and facilities: results of the European Heart Rhythm Association Survey.Europace 2014;16(8):1236-9. doi: 10.1093/ europace/euu209.

4. Naccarelli G V, Conti JB, Dimarco JP, Tracy CM. Heart Rhythm Society. Task force 6: training in specialized electro-physiology, cardiac pacing, and arrhythmia management endorsed by the Heart Rhythm Society.J Am Coll Cardiol. 2008; 22;51(3): 374-80. doi: 10.1016/j.jacc.2007.11.014

5. Merino Jl, Arribas F, Botto Gl, Huikuri H, Kraemer LI, Linde C, et al. Core curriculum for the heart rhythm specialist. Europace 2009; 11 Suppl 3:iii1-26. doi: 10.1093/europace/eup215

6. Varela G, Tortajada G, Olaizola S, Reyes Caorsi W. Implante de marcapaso biventricular por vena cava superior izquierda persistente [Resumen]. Rev Urug Cardiol. 2016; 31 Supl 1:314.
7. Pombo Jiménez M, Cano Pérez O, Lorente Carreño D, Chimeno García J. Spanish Pacemaker Registry. Registro español de marcapasos. 15th Official report of the Spanish Society of Cardiology Working Group on Cardiac Pacing (2017). Rev Esp Cardiol. (Engl Ed). 2018;71(12):1059-68. doi: 10.1016/j. rec.2018.08.015.

8. Danish pacemaker and ICD register annual report [Internet]. Odence: Odence University Hospital; 2016. [consulta 30 Ago 2019]. Disponible en: https://ssl.icddata.dk/download/Danish_Pacemaker_and_ICD_Register_Annual_Report_2016.pdf

9. Mond HG, Proclemer A. The 11th world survey of cardiac pacing and implantable cardioverter-defibrillators: calendar year 2009-a World Society of Arrhythmia's Project. Pacing Clin Electrophysiol. 2011;34(8):1013-27. doi: 10.1111/j.1540-8159.2011. 03150.x

10. Gupta N, Kiley ML, Anthony F, Young C, Brar S, Kwaku K. Multi-center, community-based cardiac implantable electronic devices registry: population, device utilization, and outcomes. J Am Heart Assoc. 2016; 5(3):e002798. doi: 10.1161/JAHA.115.002798

11. Gadler F, Valzania C, Linde C. Current use of implantable electrical devices in Sweden: data from the Swedish pacemaker and implantable cardioverter-defibrillator registry. Europace 2015; 17(1): 69-77. doi:10.1093/europace/euu233

12. Hettiarachchi EMMS, Arsene C, Fares S, Faraj A, Saulitis E, Losito S, et al. Fluoroscopy-guided axillary vein puncture, a reliable method to prevent acute complications associated with pacemaker, defibrillator, and cardiac resynchronization therapy leads insertion. J Cardiovasc Dis Diagn. 2014; 2 (1):1-5. doi:10.4172/2329-9517.1000136

13. Poole JE, Gleva MJ, Mela T, Chung MK, Uslan DZ, Borge R, et al. Complication rates associated with pacemaker or implantable cardioverter-defibrillator generator replacements and upgrade procedures: results from the REPLACE registry. Circulation 2010;122(16): 1553-61. doi: 10.1161/CIRCULATIONAHA.110.976076

14. Munawar DA, Mahajan R, Linz D, Wong GR, Khokhar KB, Thiyagarajah A, et at. Predicted longevity of contemporary cardiac implantable electronic devices: a call for industry-wide "Standardized" reporting. Heart Rhythm. 2018;15(12):1756-63. doi: 10.1016/j.hrthm.2018.07.029 